\title{
Separability of Rank Two Quantum States
}

\author{
Sergio Albeverio [, Shao-Ming Fei [2, Debashish Goswami [ \\ Institut für Angewandte Mathematik, Universität Bonn, D-53115, Bonn.
}

\begin{abstract}
Explicit sufficient and necessary conditions for separability of higher dimensional quantum systems with rank two density matrices are given. A nonseparability inequality is also presented, for the case where one of the eigenvectors corresponding to nonzero eigenvalues is a maximally entangled state.
\end{abstract}

PACS numbers: 03.65.Bz, 89.70.+c

\footnotetext{
${ }^{1}$ SFB 256; SFB 237; BiBoS; CERFIM(Locarno); Acc. Arch.; USI(Mendriso) e-mail: albeverio@uni-bonn.de

${ }^{2}$ Institute of Applied Mathematics, Chinese Academy of Science, Beijing e-mail: fei@uni-bonn.de

${ }^{3}$ Alexander von Humboldt Fellow

e-mail: goswamid@wiener.iam.uni-bonn.de
} 
Quantum entanglement has been investigated for decades because of its importance in the foundations of quantum mechanics, particularly in relation with quantum nonseparability and the violation of Bell's inequalities [1]. Recently it has been viewed also as a potentially useful resource for communication, information processing and quantum computing [2], such as for the investigation of quantum teleportation [3, 4], dense coding [5], decoherence in quantum computers and the evaluation of quantum cryptographic schemes [6.

Due to recent works by Peres [7] and Horodecki et al [8] there exist a simple criterion allowing one to judge, whether a given density matrix $\rho$, representing a $2 \times 2$ or $2 \times 3$ composite system, is separable. Nevertheless, the general problem of finding sufficient and necessary conditions for separability in higher dimensions remains open (see e.g. [9, 10] and references therein).

A general condition for separability of a quantum state could in principle be obtained from the measure of entanglement. To quantify the degree of entanglement a number of entanglement measures, such as the entanglement of formation and distillation [11, 12, 13], negativity [7, 14] and relative entropy [13, 15], have been proposed for bipartite states [6,8,15-18]. However most proposed measures of entanglement involve extremizations which are difficult to handle analytically. For instance, the "entanglement of formation" [11], which is intended to quantify the amount of quantum communication required to create a given state, is defined for arbitrary dimension, but so far no explicit analytic formulae for entanglement of formation have been found for systems larger than a pair of qubits (spin- $\frac{1}{2}$ particles). For the case of a pair of qubits, it has been shown that the entanglement of formation can be expressed as a monotonically increasing function of the "concurrence" variable $C$. This function ranges from 0 to 1 as $C$ goes from 0 to 1 , so that one can take the concurrence as a measure of entanglement in its own right [17].

In this letter, we introduce a generalized concurrence and study sufficient and necessary conditions for separability of higher dimensional quantum systems. In particular, we consider density matrices with rank two. The separability condition for these kind of mixed states in arbitrary dimensions is explicitly given. In addition, we present a non separability inequality valid in the case where one of the eigenvectors, corresponding to nonzero 
eigenvalues, of a density matrix is a maximally entangled state.

We first introduce a generalized concurrence $C_{N}$ for $N$-dimensional quantum states. Let $H$ be an $N$-dimensional complex Hilbert space, with $e_{i}, i=1, \ldots, N$, as an orthonormal basis. A general pure state on $H \otimes H$ is of the form,

$$
|\Psi\rangle=\sum_{i, j=1}^{N} a_{i j} e_{i} \otimes e_{j}, \quad a_{i j} \in \mathbb{C}
$$

with the normalization $\sum_{i, j=1}^{N} a_{i j} a_{i j}^{*}=1$ (* denoting complex conjugation).

Let $U$ denote a unitary transformation on the Hilbert space $\mathcal{H}$, such that

$$
U e_{i} \mapsto \sum_{j=1}^{N} b_{i j} e_{j}, \quad b_{i j} \in \mathbb{C}
$$

and $\sum_{j=1}^{N} b_{i j} b_{k j}^{*}=\delta_{i k}$ (with $\delta_{i k}$ the usual Kronecker's symbol). We call a quantity an invariant associated with the state $|\Psi\rangle$ if it is invariant under all local unitary transformations, i.e. all maps from $H \otimes H$ to itself of the form $U \otimes U$. Let $A$ denote the matrix given by $(A)_{i j}=a_{i j}$. By generalizing the results of analysis on invariants for qubits [19], we can show that the following quantities are invariants under local unitary transformations:

$$
I_{\alpha}=\operatorname{Tr}\left(A A^{\dagger}\right)^{\alpha+1}, \quad \alpha=0,1, \ldots, N-1
$$

(with $A^{\dagger}$ the adjoint of the matrix $A$ ).

We define the generalized concurrence to be:

$$
C_{N}=\sqrt{\frac{N}{N-1}\left(I_{0}^{2}-I_{1}\right)}=\sqrt{\frac{N}{2(N-1)} \sum_{i, j, k, m=1}^{N}\left|a_{i k} a_{j m}-a_{i m} a_{j k}\right|^{2}} .
$$

For $N=2$ we have $C_{2}=2\left|a_{11} a_{22}-a_{12} a_{21}\right|$, which is just the definition of concurrence for a pure state of two qubits 17 .

For general $N$, we see that when the state $|\Psi\rangle$ is factorizable in the sense that $a_{i j}=a_{i} b_{j}$ for some $a_{i}, b_{j} \in \mathbb{C}, i=1, \ldots, N, C_{N}$ is zero. When $|\Psi\rangle$ is maximally entangled, $\left|a_{i i}\right|=$ $1 / \sqrt{N}, a_{i j}=0$ for $i \neq j, C_{N}$ is one. In terms of the Schmidt decomposition, a given $|\Psi\rangle$ can always be written in the form, possibly by changing the orthonormal basis $\left\{e_{i}\right\}, i=1, \ldots, N$ :

$$
|\Psi\rangle=\sum_{i=1}^{N} \sqrt{\Lambda_{i}} e_{i} \otimes e_{i}
$$


where $\sum_{i=1}^{N} \Lambda_{i}=1, \Lambda_{i} \geq 0$. The invariants are then of the form

$$
I_{\alpha}=\sum_{i=1}^{N} \Lambda_{i}^{\alpha+1}, \quad \alpha=0, \ldots, N-1
$$

We note that

$$
I_{1}=\sum_{i=1}^{N} \Lambda_{i}^{2}=I_{0}^{2}-\sum_{i \neq j}^{N} \Lambda_{i} \Lambda_{j}
$$

Therefore $C_{N}=0$ implies that $\sum_{i \neq j}^{N} \Lambda_{i} \Lambda_{j}=0$. As $\Lambda_{i} \geq 0$ and $\sum_{i=1}^{N} \Lambda_{i}=1$, we have that in this case only one of the $\Lambda_{i}, i=1, \ldots N$ is equal to 1 and all other ones are zero. Hence $C_{N}=0$ implies that $|\Psi\rangle$ is separable.

If $C_{N}=1$, we have $\sum_{i \neq j}^{N} \Lambda_{i} \Lambda_{j}=\frac{N-1}{N}$, which is equivalent to the condition $\sum_{i=1}^{N} \Lambda_{i}^{2}=1 / N$, according to the normalization $\sum_{i=1}^{N} \Lambda_{i}=1$. The equation $\sum_{i=1}^{N} \Lambda_{i}^{2}=1 / N$ describes an $(N-1)$ dimensional sphere in $\mathbb{R}^{N}$ with radius $1 / \sqrt{N}$, whereas $\sum_{i=1}^{N} \Lambda_{i}=1$ is a hyperplane in $\mathbb{R}^{N}$. These hypersurfaces have only one contact point at $\Lambda_{i}=1 / N, i=1, \ldots, N$. Therefore $C_{N}=1$ implies that $|\Psi\rangle$ is maximally entangled. We remark that the above properties of $C_{N}$ do not mean that $C_{N}$ is in general a suitable measure for general $N$-dimensional bipartite quantum pure states. It can however be shown that when the matrix $A A^{\dagger}$ has only two different nonzero eigenvalues, the entanglement of formation is a monotonically increasing function of $C_{N}$, thus $C_{N}$ can indeed be interpreted as a measure of entanglement in this case.

Let $\rho$ be a rank two state in $H \otimes H$, with $\left|E_{1}\right\rangle,\left|E_{2}\right\rangle$ being its two orthonormal eigenvectors corresponding to the two nonzero eigenvalues:

$$
\rho=p\left|E_{1}\right\rangle\left\langle E_{1}|+q| E_{2}\right\rangle\left\langle E_{2}\right|
$$

where $q=1-p \in(0,1)$. Generally $\left|E_{k}\right\rangle=\sum_{i, j=1}^{N} a_{i j}^{k} e_{i} \otimes e_{j}, a_{i j}^{k} \in \mathbb{C}$, with normalization $\sum_{i, j=1}^{N} a_{i j}^{k}\left(a_{i j}^{k}\right)^{*}=1, k=1,2$. We would like to give an explicit algebraic condition for the separability of the above state in terms of $a_{i j}^{k}, k=1,2$.

With the notations:

$$
\begin{aligned}
& \alpha_{i j}^{k l}=a_{i j}^{2} a_{k l}^{2}-a_{i l}^{2} a_{k j}^{2}, \quad \gamma_{i j}^{k l}=a_{i j}^{1} a_{k l}^{1}-a_{i l}^{1} a_{k j}^{1} \\
& \beta_{i j}^{k l}=a_{i j}^{1} a_{k l}^{2}+a_{i j}^{2} a_{k l}^{1}-a_{i l}^{2} a_{k j}^{1}-a_{i l}^{1} a_{k j}^{2},
\end{aligned}
$$


we have the following conclusion:

[Theorem 1]. If all $a_{i j}^{k}$ are real, $\rho$ is separable if and only if one of the following quantities $\left(\Delta_{1}\right.$ or $\left.\Delta_{2}\right)$ is zero :

$$
\begin{gathered}
\Delta_{1}=\sum_{i j k l}\left|\gamma_{i j}^{k l}-\left(1-p^{-1}\right) \alpha_{i j}^{k l}\right|^{2}+\sum_{i j k l m n}\left|\beta_{i j}^{k l} \alpha_{m n}^{k l}-\alpha_{i j}^{k l} \beta_{m n}^{k l}\right|^{2} ; \\
\Delta_{2}=\sum_{i j k l}\left|\gamma_{i j}^{k l}+\left(1-p^{-1}\right) \alpha_{i j}^{k l}\right|^{2}+\sum_{i j k l}\left|\beta_{i j}^{k l}\right|^{2} ;
\end{gathered}
$$

or equivalently one of the following two sets of relations $((6)$ or $(7))$ hold:

$$
\begin{gathered}
\gamma_{i j}^{k l}=\left(1-p^{-1}\right) \alpha_{i j}^{k l}, \quad \beta_{i j}^{k l} \alpha_{m n}^{k l}=\alpha_{i j}^{k l} \beta_{m n}^{k l}, \quad \forall i, j, k, l, m, n . \\
\gamma_{i j}^{k l}=-\left(1-p^{-1}\right) \alpha_{i j}^{k l}, \quad \beta_{i j}^{k l}=0, \quad \forall i, j, k, l .
\end{gathered}
$$

[Proof]. From the discussions above we have that a state $|\Psi\rangle=\sum_{i, j=1}^{N} a_{i j} e_{i} \otimes e_{j}$ is separable if and only if the generalized concurrence $C_{N}$ is zero, i.e.,

$$
a_{i j} a_{k l}=a_{i l} a_{k j}, \quad \forall i, j, k, l \text {. }
$$

Therefore a vector of the form $\left|E_{1}\right\rangle+\lambda\left|E_{2}\right\rangle, \lambda \in \mathbb{C}$ is separable if and only if $\lambda$ is a common root of the following equation set $E q_{i j ; k l}$ :

$$
\alpha_{i j}^{k l} \lambda^{2}+\beta_{i j}^{k l} \lambda+\gamma_{i j}^{k l}=0, \quad \forall i, j, k, l
$$

We first prove the necessity part of the theorem. Suppose that $\rho=\sum_{t=1}^{l} p_{t}^{\prime}\left|U_{t}\right\rangle\left\langle U_{t}\right|$, with $l$ some positive integer and $0<p_{t}^{\prime}<1, \sum p_{t}^{\prime}=1$, gives a decomposition of $\rho$ in terms of separable normalized vectors $U_{t}$ (where $\left|U_{t}\right\rangle\left\langle U_{t}\right|$ means the projection onto the vector $U_{t}$ ). As each of these vectors must be in the range of $\rho$ (since for each fixed $t,\left|U_{t}\right\rangle\left\langle U_{t}\right| \leq p_{t}^{\prime-1} \rho$ ), we can write them as linear combinations of the two eigenvectors $\left|E_{1}\right\rangle$ and $\left|E_{2}\right\rangle$ which span the range of $\rho$. So let us assume that $U_{t}=c_{1}^{t}\left|E_{1}\right\rangle+c_{2}^{t}\left|E_{2}\right\rangle$ (for some $c_{1}^{t}, c_{2}^{t} \in \mathbb{C}$ ). We deal with the problem in two cases:

Case 1. $\left|E_{2}\right\rangle$ is not separable

In this case, since $c_{1}^{t}$ can not be $0, E_{1}+\lambda_{t} E_{2}$ will be a separable vector, where $\lambda_{t}=c_{2}^{t} / c_{1}^{t}$. Clearly, not all $\lambda_{t} \mathrm{~s}$ can be equal, otherwise all the $U_{t} \mathrm{~s}$ would be constant multiples of a fixed vector, and $\rho$ would be of rank 1 . Hence there must be at least two distinct choices of 
$\lambda_{t}$. On the other hand, as $\left|E_{2}\right\rangle$ is not separable, its generalized concurrence $C_{N}$ is not zero. Hence there is some $i_{0}, j_{0}, k_{0}, l_{0}$ such that $\alpha_{i_{0} j_{0}}^{k_{0} l_{0}} \neq 0$, i.e. the relation $E q_{i_{0} j_{0} ; k_{0} l_{0}}$ is indeed a quadratic equation. It must have exactly two roots, say $\lambda^{(1)}, \lambda^{(2)}$, and so these two values are the only possible choices for the $\lambda_{t}$ 's. But in order that there is not only one possible choice, the above two roots must be different. And all the relations $E q_{i j ; k l}$ have these different two roots. Consider for any $i, j, k, l$,

Situation 1: If $\alpha_{i j}^{k l} \neq 0$, the corresponding relation (9) is then not an identity. All the quadratic equations in the equation set $E q_{i j ; k l}$ have the same two distinct roots. In other words, $E q_{i j ; k l}$ and $E q_{i_{0} j_{0} ; k_{0} l_{0}}$ have the same roots. From the standard theory of quadratic equations, we have

$$
\begin{aligned}
\beta_{i j}^{k l} \alpha_{i_{0} j_{0}}^{k_{0} l_{0}} & =\beta_{i_{0} j_{0}}^{k_{0} l_{0}} \alpha_{i j}^{k l}, \\
\gamma_{i j}^{k l} \alpha_{i_{0} j_{0}}^{k_{0} l_{0}} & =\gamma_{i_{0} j_{0}}^{k_{0} l_{0}} \alpha_{i j}^{k l} .
\end{aligned}
$$

Situation 2 : If $\alpha_{i j}^{k l}=0$, then the equations $E q_{i j ; k l}$ become identities, i.e. $\beta_{i j}^{k l}$ and $\gamma_{i j}^{k l}$ must be 0 too, because otherwise at least one of the relations $E q_{i j ; k l}$ would be a linear equation, and so could not be satisfied by two distinct roots. Thus in this case (10) and (11) also hold (as both sides in both equations vanish).

Thus we have obtained (10) and (11) for arbitrary $i j k l$ and for all $i_{0} j_{0} k_{0} l_{0}$ such that $\alpha_{i_{0} j_{0}}^{k_{0} l_{0}} \neq 0$. As (10) and (11) are trivially valid whenever $i j k l$ and $i_{0} j_{0} k_{0} l_{0}$ are such that $\alpha_{i j}^{k l}=\alpha_{i_{0} j_{0}}^{k_{0} l_{0}}=0$, we have indeed established (10) and (11) for all quadruplets $i j k l, i_{0} j_{0} k_{0} l_{0}$.

We now denote the above two distinct roots, which are common to all of the equations $E q_{i j ; k l}$, by $\mu_{1}, \mu_{2}$, with the convention that in case the roots are real, $\mu_{2}$ will denote the larger one. Each vector $U_{t}$ is either of the form $\tilde{E}_{1}=\frac{E_{1}+\mu_{1} E_{2}}{\sqrt{1+\left|\mu_{1}\right|^{2}}}$ or of the form $\tilde{E}_{2}=\frac{E_{1}+\mu_{2} E_{2}}{\sqrt{1+\left|\mu_{2}\right|^{2}}}$. Therefore we can write $\rho$ as, $\rho=p^{\prime}\left|\tilde{E}_{1}\right\rangle\left\langle\tilde{E}_{2}\left|+\left(1-p^{\prime}\right)\right| \tilde{E}_{2}\right\rangle\left\langle\tilde{E}_{2}\right|$, with $0<p^{\prime}<1$. Comparing with the coefficients of $\left|E_{k}\right\rangle\left\langle E_{l}\right|, k, l=1,2$ in the expression (5), we get that the above decomposition of $\rho$ is equivalent to the following two relations:

$$
\frac{p^{\prime}}{1+\left|\mu_{1}\right|^{2}}+\frac{1-p^{\prime}}{1+\left|\mu_{2}\right|^{2}}=p, \quad \frac{\mu_{1} p^{\prime}}{1+\left|\mu_{1}\right|^{2}}+\frac{\mu_{2}\left(1-p^{\prime}\right)}{1+\left|\mu_{2}\right|^{2}}=0 .
$$

Solving (12) for $p$ and $p^{\prime}$ we get

$$
p=\left(1-\mu_{1} \mu_{2} \frac{\bar{z}}{z}\right)^{-1}, \quad p^{\prime}=\frac{\mu_{2}\left(1+\left|\mu_{1}\right|^{2}\right)}{z-\mu_{1} \mu_{2} \bar{z}},
$$


where $z=\mu_{2}-\mu_{1}$.

As $a_{i j}^{k}$ s are real numbers, $\mu_{1}, \mu_{2}$ are roots of a quadratic equation with real coefficients. For a quadratic equation $a x^{2}+b x+c=0$ with $a, b, c \in \mathbb{R}, a \neq 0$, and roots $\alpha, \beta$ with $\alpha \neq \beta$, we have that $\frac{\bar{\alpha}-\bar{\beta}}{\alpha-\beta}$ is either +1 or -1 , depending on whether $b^{2}-4 a c$ is positive or negative respectively. Hence in our case we have that $\mu_{1} \mu_{2}=+\left(1-p^{-1}\right)$ or $-\left(1-p^{-1}\right)$. On the other hand, since $\mu_{1} \mu_{2}$ is real, the solution for $p^{\prime}$ in (13) implies that $\frac{\mu_{2}}{\mu_{2}-\mu_{1}}$ is real, which is possible if and only if either the roots are both real or the roots are both purely imaginary.

For the the case where the roots are both purely imaginary, we have, $\mu_{1} \mu_{2}=-\left(1-p^{-1}\right)$; and a direct simplification from (13) gives $p^{\prime}=\frac{1}{2}$. The condition for having purely imaginary roots of quadratic equations gives that $\beta_{i j}^{k l}=0, \forall i j k l$.

In the case where the roots are real, $\mu_{2}$ being the larger root by assumption, we have $\mu_{1} \mu_{2}=\left(1-p^{-1}\right)$. By replacing $\left|\mu_{1}\right|^{2}$ by $\mu_{1}^{2}$ in the expression of $p^{\prime}$ in (13), we get that the condition that $p^{\prime}$ takes values between 0 and 1 is equivalent to $\mu_{2}>0, \mu_{1}<0$, which is trivially valid since $\mu_{2}>\mu_{1}$ and $\mu_{1} \mu_{2}<0$ in this case.

It remains to observe case that $\mu_{1} \mu_{2}$ is nothing but the ratio $\frac{\gamma_{i_{0} j_{0}}^{k_{0} l_{0}}}{\alpha_{i_{0} j_{0}} l_{0}}$ which is either $1-p^{-1}$ or $-\left(1-p^{-1}\right)$. Taking into account relation (11), one concludes that either $\gamma_{i j}^{k l}=\left(1-p^{-1}\right) \alpha_{i j}^{k l}$ or $\gamma_{i j}^{k l}=-\left(1-p^{-1}\right) \alpha_{i j}^{k l}$ for all $i, j, k, l$. It then follows that in the second case $\beta_{i j}^{k l}=0$ for all $i, j, k, l$. This completes the proof for the necessity part in the case 1 .

Case 2. $\left|E_{2}\right\rangle$ is separable

In this case from (8) we have $\alpha_{i j}^{k l}=0, \forall i, j, k, l$. Since not all of the $U_{t}$ 's can be multiples of $\left|E_{2}\right\rangle$, we must have at least one choice of $\lambda$ such that $E_{1}+\lambda E_{2}$ is separable. This $\lambda$ must be a common root to all equations $E q_{i j ; k l}$ as before. All these equations are now linear ones. Excluding the trivial case that all of them are identically 0 (which means that $\left|E_{1}\right\rangle$ is also separable), we see that there is only one possible choice of $\lambda$ (which is the solution to a nontrivial linear equation). Then $\rho$ can be expressed as $\rho=p^{\prime \prime}\left|E_{2}\right\rangle\left\langle E_{2}\right|+(1-$ $\left.p^{\prime \prime}\right) \frac{\left|E_{1}+\lambda E_{2}\right\rangle\left\langle E_{1}+\lambda E_{2}\right|}{1+|\lambda|^{2}}$. That is $p^{\prime \prime}=1$, which is clearly a contradiction. Thus in case where $\left|E_{2}\right\rangle$ is separable, we must have $\left|E_{1}\right\rangle$ to be separable too in order for $\rho$ to be separable. It is clear that in this case the conditions of the theorem hold. 
Now we prove the sufficiency part for the theorem. If either (6) or (7) holds, from the previous analysis it is clear that the equations $E q_{i j ; k l}$ have common roots. If $\left|E_{2}\right\rangle$ is not separable, then not all of these equations are identities. And there are at most two common roots. Furthermore, if (6) holds, the product of the two roots must be $\left(1-p^{-1}\right)<0$, so that the two roots be real and unequal, with opposite signs. Denoting them by $\mu_{1}, \mu_{2}$ as before, we can easily get from the arguments given earlier an explicit decomposition of $\rho$ in terms of separable pure states. Similarly, if (7) holds, the two roots must be purely imaginary (since $\beta_{i j}^{k l}=0$ ) and the rest also follows easily from our earlier analysis. Finally, if $\left|E_{2}\right\rangle$ is separable, either of the conditions (6) or (7) forces $\gamma_{i j}^{k l}$ to be 0 for all $i j k l$. Hence $\left|E_{1}\right\rangle$ is separable too and $\rho$ is trivially separable. This completes the proof.

The conclusions in Theorem 1 can easily be generalized to the complex case, $a_{i j}^{k} \in \mathbb{C}$. [Theorem 2]. $\rho$ is separable if and only if there is $\theta \in \mathbb{R}$ such that

$$
\begin{gathered}
\gamma_{i j}^{k l}=e^{i \theta}\left(1-p^{-1}\right) \alpha_{i j}^{k l}, \\
\beta_{i j}^{k l} \alpha_{m n}^{k l}=\alpha_{i j}^{k l} \beta_{m n}^{k l}, \quad \forall i, j, k, l, m, n ;
\end{gathered}
$$

and

$$
\frac{\mu_{2}\left(1+\left|\mu_{1}\right|^{2}\right)}{z-\mu_{1} \mu_{2} \bar{z}} \in[0,1]
$$

where $z=e^{i \theta} \bar{z}, z=\mu_{2}-\mu_{1} \neq 0, \mu_{1}$ and $\mu_{2}$ are the roots of the equation $\alpha_{i j}^{k l} \lambda^{2}+\beta_{i j}^{k l} \lambda+\gamma_{i j}^{k l}=0$, for some $i, j, k, l$ such that $\alpha_{i j}^{k l} \neq 0$.

The proof for the necessity part of the theorem is similar to the proof of the corresponding part in Theorem 1. One only needs to note that since $z / \bar{z}$ is of modulus 1 , and of the form $e^{i \theta}$ for some $\theta$. From the relations (13), we obtain then that $\frac{\mu_{2}\left(1+\left|\mu_{1}\right|^{2}\right)}{z-\mu_{1} \mu_{2} \bar{z}}$ is a real number between 0 and 1 .

The proof of the sufficiency part for the theorem essentially follows by observing that the arguments given in the necessity part can be reversed. From the previous analysis, it follows from (14) and (15) that the equations $E q_{i j ; k l}$ have common roots. In case all $\alpha_{i j}^{k l}$ are 0 , by the condition $\gamma_{i j}^{k l}=e^{i \theta}\left(1-p^{-1}\right) \alpha_{i j}^{k l}$, we see that all $\gamma_{i j}^{k l}$ are 0 too. Hence both $\left|E_{1}\right\rangle$ and $\left|E_{2}\right\rangle$ are separable, and so is $\rho$. In the other situations where some of the $\alpha_{i j}^{k l}$ 's are nonzero, the corresponding equations $E q_{i j ; k l}$ have exactly two roots which are different by the condition (16). Moreover, (16) ensures that the explicit solution for the choice of 
$p^{\prime}$, which gives the decomposition of $\rho$ in terms of separable states, is indeed a valid choice between 0 and 1 . This completes the proof of theorem 2 .

[Corollary]. Let $\left|E_{2}\right\rangle$ be the maximally entangled vector given by $\left|E_{2}\right\rangle=\frac{1}{\sqrt{N}} \sum_{i=1}^{N} e_{i} \otimes e_{i}$. For any vector $\left|E_{1}\right\rangle$ which is orthogonal to $\left|E_{2}\right\rangle, \rho=p\left|E_{1}\right\rangle\left\langle E_{1}|+(1-p)| E_{2}\right\rangle\left\langle E_{2}\right|$ is not separable for $0<p<\frac{1}{2}$.

[Proof]. From the necessary condition for separability, $\gamma_{i j}^{k l}=e^{i \theta}\left(1-p^{-1}\right) \alpha_{i j}^{k l}$, we get that $C_{(1)}=\frac{1-p}{p} C_{(2)}$, where $C_{(1)}=\sqrt{\frac{N}{2(N-1)} \sum_{i, j, k, m=1}^{N}\left|\gamma_{i j}^{k l}\right|^{2}}$ and $C_{(2)}=\sqrt{\frac{N}{2(N-1)} \sum_{i, j, k, m=1}^{N}\left|\alpha_{i j}^{k l}\right|^{2}}$ are the generalized concurrences associated with the states $\left|E_{1}\right\rangle$ and $\left|E_{2}\right\rangle$ respectively. As a pure state is separable if and only if the corresponding generalized concurrence is zero, a necessary condition for separability is that $C_{(1)}$ and $C_{(2)}$ should be inversely proportional to the contribution of the corresponding pure state to $\rho$, i.e. the eigenvalue corresponding to that eigenstate. From $\frac{C_{(1)}}{C_{(2)}}=\frac{1-p}{p} \leq 1$, we have $p \geq \frac{1}{2}$.

We have studied the sufficient and necessary conditions for separability of higher dimensional quantum systems of density matrices with rank two, in terms of the generalized concurrence. Our approach concerns the eigenstates of a given density matrix $\rho$ (thus results as in our corollary are straightforward to deduce). It gives a direct minimal decomposition of separable states in terms of pure product states. The method can also be used to check whether it is possible to construct a separable mixed state from two given pure states.

Another elegant approach, based on the positive partial transpose (PPT) condition, dealing with the sufficient and necessary criteria for separability of higher dimensional quantum systems was presented in 20] tor a given density matrix $\rho$, one can judge its separabilty by calculating its supporting dimensions ( $\rho$ is supported on $M \times N$ if this is the smallest product Hilbert space on which $\rho$ can act), checking the positiveness of partial transposed $\rho$ and comparing the rank of $\rho$ with dimensions $M, N$.

\section{Acknowledgement:}

One of the authors (D. Goswami) would like to thank Prof. K. R. Parthasarathy from I.S.I. (New Delhi, India) for his motivating lecture-series on quantum codes, quantum computation and many related topics delivered in the academic year 1999-2000. He would also

\footnotetext{
${ }^{4}$ We would like to thank the referee for introducing this paper to us.
} 
express his gratefulness to Prof. S. Albeverio for inviting him to I.A.M. (Bonn) and the Av. Humboldt Foundation for the research fellowship.

\section{References}

[1] J. Bell, Physics 1 (1964) 195.

[2] See, for example, D.P. DiVincenzo, Science 270, 255 (1995).

[3] C.H. Bennett, G. Brassard, C. Crépeau, R. Jozsa, A. Peres, and W.K. Wootters, Phys.Rev.Lett. 70, 1895 (1993).

[4] S. Albeverio and S.M. Fei, Phys.Lett. A 276(2000)8-11.

[5] C.H. Bennett and S.J. Wiesner, Phys.Rev.Lett. 69, 2881 (1992).

[6] See, for example, C.A. Fuchs, N. Gisin, R.B. Griffiths, C-S. Niu, and A. Peres, Phys.Rev.A, 56, 1163 (1997) and references therein.

[7] A. Peres, Phys. Rev. Lett. 77, 1413 (1996).

[8] M. Horodecki, P. Horodecki, and R. Horodecki, Phys. Lett. A 223, 1 (1996).

[9] M. Lewenstein, D. Bruss, J. I. Cirac, B. Kraus, M. Kuś, J. Samsonowicz, A. Sanpera, and R. Tarrach, J. Mod. Opt. 47, 2481 (2000).

[10] M. Horodecki, P. Horodecki, and R. Horodecki, preprint LANL quant-ph/0006071

[11] C.H. Bennett, D.P. DiVincenzo, J.A. Smolin, and W.K. Wootters, Phys. Rev. A 54, 3824 (1996).

[12] C.H. Bennett, H.J. Bernstein, S. Popescu, and B. Schumacher, Phys. Rev. A 53, 2046 (1996).

[13] V. Vedral, M.B. Plenio, M.A. Rippin, and P.L. Knight,Phys. Rev. Lett. 78, 2275 (1997);

V. Vedral, M.B. Plenio, K. Jacobs, and P.L. Knight, Phys. Rev. A 56, 4452 (1997);

V. Vedral and M.B. Plenio, Phys. Rev. A 57, 1619 (1998). 
[14] K. Życzkowski and P. Horodecki, Phys. Rev. A 58, 883 (1998).

[15] B. Schumacher and M.D. Westmoreland, Relative entropy in quantum information theory, quant-ph/0004045.

[16] M. Horodecki, P. Horodecki, and R. Horodecki, Phys. Rev. Lett. 80, 5239 (1998).

[17] S. Hill and W.K. Wootters, Phys. Rev. Lett. 78, 5022 (1997).

W.K. Wootters, Phys. Rev. Lett. 80, 2245 (1998).

[18] R.F. Werner and M.M. Wolf,Phys. Rev. A 61, 062102 (2000).

[19] N. Linden and S. Popescu, Fortsch. Phys. 46, 567-578 (1998).

[20] P. Horodecki, M. Lewenstein, G. Vidal and I. Cirac, Phys. Rev. A 62, 032310 (2000). 